\title{
Diffusive and precessional spin dynamics in a two-dimensional electron gas with disorder: a gauge theory view
}

\author{
I.V. Tokatly ${ }^{1,2,4}$ and E. Ya. Sherman ${ }^{3,4}$ \\ 1 European Theoretical Spectroscopy Facility (ETSF), \\ Departamento de Fisica de Materiales, \\ Universidad del País Vasco UPV/EHU and Centro \\ Mixto CSIC-UPV/EHU, San Sebastian, Spain \\ 2 Moscow Institute of Electronic Technology, Zelenograd, 124498 Russia \\ 3 Department of Physical Chemistry, \\ Universidad del País Vasco UPV/EHU, 48080 Bilbao, Spain \\ 4 IKERBASQUE Basque Foundation for Science, \\ Alameda Urquijo 36-5, 48011, Bilbao, Bizkaia, Spain
}

\begin{abstract}
We develop a gauge theory for diffusive and precessional spin dynamics in two-dimensional electron gas with disorder. Our approach reveals a direct connections between the absence of the equilibrium spin current and strong anisotropy in the spin relaxation: both effects arise if the spinorbit coupling is reduced to a pure gauge $S U(2)$ field. In this case, by a gauge transformation in the form of a local $S U(2)$ rotation in the spin subspace the spin-orbit coupling can be removed. The resulting spin dynamics is exactly described in terms of two kinetic coefficients: the spin diffusion and electron mobility. After the inverse transformation, full diffusive and precessional spin density dynamics, including the anisotropic spin relaxation, formation of stable spin structures, and spin precession induced by a macroscopic current, is restored. Explicit solutions of the spin evolution equations are found for the initially uniform spin density and for stable nonuniform structures. Our analysis demonstrates a universal relation between the spin relaxation rate and spin diffusion coefficient.
\end{abstract}

PACS numbers: 72.25.-b 


\section{INTRODUCTION.}

Description of the spin dynamics of a two-dimensional electron gas is one of the most important problems for fundamental and applied modern spintronics. [1-3] Two mutually related problems in this field attract a great deal of attention: spin current and spin relaxation. Spin currents describe how the spin density pattern changes with time mainly due to the spin transfer between different parts of the electron gas. Since the spin dynamics of interest occurs usually in systems out of equilibrium, spin relaxation becomes important and contributes strongly into the evolution of spin density pattern.

The key for understanding these properties is the spin-orbit coupling, making the orbital and spin degrees of freedom mutually dependent. Spin-orbit coupling has many crucial influences on the properties of the systems where it occurs: the typical examples are nuclei, elementary particles, atoms, and electrons in solids. Spin-orbit coupling, in general, makes the spin a non-conserved quantity, thus leading to a spin relaxation. It causes mutually dependent spin, charge, and mass flows in solids and quantum liquids. [4 6] In addition, spin-orbit coupling leads to a spin response to an external electric field, providing an ability of spin manipulation by the electric field driving the dynamics in the orbital degrees of freedom.[7]

The general techniques for calculation of spin relaxation and spin current out of equilibrium are the classical or quantum Boltzmann-like equation for the spin-density matrix [8-12] and nonequilibrium Green functions.[13, 14] In this approach, the description of the electron dynamics takes into account possible relaxation processes due to the presence of spin-orbit coupling, disorder, phonons, and electron-electron collisions. The experimentally observable spin dynamics is due to the spin-orbit (spin-momentum) coupling. At the equilibrium, the expectation value of the spin current can be calculated directly. Surprisingly, such a direct calculation demonstrated that the spin current can exist even in the equilibrium state of a two-dimensional electron gas with spin-orbit coupling. [15] This observation brought a puzzle for the understanding of the basic phenomena in spin transport since the equilibrium spin current is not related to any spatial spin accumulation that can be seen experimentally.

On the other hand, the spin dynamics due to the spin-orbit coupling can be understood in terms of a theory where the coupling is treated as a non-Abelian gauge field, and the corresponding formalism can be applied [16, 17]. On a single-electron scale, for example, for 
electrons in quantum dots, the gauge transformation of the spin-orbit field was employed in Refs. [18, 19] and used for the analysis of experimental results on spin manipulation by electric field in quantum dots in Ref.[20]. Other interesting theoretical examples of applications of the non-Abelian gauge field approach for single-electron spin transport and electrons in quantum dots, were found and studied (for example, Refs.[21]-[26]).

When applied to the two-dimensional electron gas, the approach based on a formal $S U(2)$ gauge invariance of the spin-orbit Hamiltonian (i. e. the symmetry with respect to local rotations in the spin subspace) proved that the equilibrium spin current is the diamagnetic response to the effective non-Abelian spin-orbit magnetic field. [27] If the spin-orbit field is a pure gauge and, thus, can be removed by a gauge transformation, the effective $S U(2)$ magnetic field is zero, and the equilibrium spin current vanishes.

Here we present a theory based on the gauge transformation, for spin dynamics in a twodimensional electron gas in the case when the spin-orbit field can be completely removed by such a gauge transformation. We show that the absence of the equilibrium spin current is directly related to the giant anisotropy in the spin relaxation rate, when the relaxation does not occur for certain spin directions.[28, 29] After gauging away the spin-orbit coupling, the entire nonequilibrium dynamics of a transformed spin becomes almost trivial and can be described phenomenologically exactly by only two transport coefficients which can be determined experimentally, or calculated theretically to any desired level of accuracy. The first is the spin diffusion coefficient and the second is the electron mobility required only when a constant electric field is applied. With the inverse transformation to the initial dynamical variables, we recover the full nontrivial dynamics of the physical spin, including the absence of the spin relaxation for certain spin directions, that is a strong anisotropy in the spin relaxation, stable spin configurations forming persistent spin helices, and spin precession due to a charge current in a constant external electric field. In addition, this approach allows making predictions for more general cases of spin-orbit coupling, including nonuniform spin-orbit fields. 


\section{SPIN CURRENT AND SPIN RELAXATION: THE CONVENTIONAL AP- PROACH.}

We begin with the conventional Hamiltonian of spin-orbit coupling in two-dimensional electron gas:

$$
H_{\mathrm{so}}=\frac{1}{2} \sum_{j}\left(\alpha_{j a}(\boldsymbol{\rho}) k_{j}+k_{j} \alpha_{j a}(\boldsymbol{\rho})\right) \sigma^{a},
$$

where $\alpha_{j a}$ is the coordinate-dependent spin-orbit coupling field, $k_{j}=-i \partial / \partial x_{j}$ is the momentum operator, Cartesian subscript indices $j=x, y$ correspond to the electron coordinate $\boldsymbol{\rho}=(x, y)$, and $\sigma^{a}$ are the Pauli matrices with the upper Cartesian indices corresponding to three directions $x, y, z$ in the spin subspace. We use the system of units with $\hbar \equiv 1$ and sum up over repeating indices. Interaction $H_{\mathrm{so}}$ arises in a two-dimensional electron gas from various sources. Two origins are considered as the most important. The first one, arising due to the inversion asymmetry of the crystal unit cell, is described by the Dresselhaus model. The other one is the Rashba field,[30] where the coupling originates from the macroscopic asymmetry of the structure hosting the two-dimensional electron gas.[31] Due to various physical origins, including material, structure, doping, and possible mechanical strain, numerical values of parameters $\alpha_{j a}$ vary strongly from system to system ranging from $10^{-12}$ $\mathrm{eV} \cdot \mathrm{cm}$ for $\mathrm{Si}$ - to $10^{-9} \mathrm{eV} \cdot \mathrm{cm}$ for GaAs-based structures and will not be discussed here.

For the coordinate-independent spin-orbit field $k_{j} \alpha_{j a}(\boldsymbol{\rho})=0$, and the Hamiltonian (1) can be presented as:

$$
H_{\mathrm{so}}=\sum_{j} \alpha_{j}\left(\mathbf{h}_{[j]} \cdot \boldsymbol{\sigma}\right) k_{j},
$$

where $\mathbf{h}_{[j]}$ is a unit-length vector, and $\alpha_{j}$ is the corresponding spin-orbit coupling constant for given component of momentum; its contribution to the Hamiltonian is, therefore, proportional to the spin projection onto the $\mathbf{h}_{[j]}$ axis. The coupling leads to a momentum-dependent spin splitting of the electron states. As a results, the Fermi line of the electron gas becomes spin-dependent and two Fermi lines in the electron gas appear. The $H_{\text {so }}$ Hamiltonian makes the velocity spin-dependent:

$$
v_{j}=\frac{k_{j}}{m}+i\left[H_{\mathrm{so}}, \rho_{j}\right]=\frac{k_{j}}{m}+\alpha_{j}\left(\mathbf{h}_{[j]} \cdot \boldsymbol{\sigma}\right),
$$

with $m$ being the electron effective mass. 
With the spin-dependent velocity in Eq.(3) we define the operator of the spin current in the form:

$$
J_{j}^{a}=\frac{1}{2} \sum_{\mathbf{k}} C_{\mathbf{k}}^{\dagger}\left(v_{j} \tau^{a}+\tau^{a} v_{j}\right) C_{\mathbf{k}},
$$

where the $S U(2)$ group generators $\tau^{a}=\sigma^{a} / 2$, and $C_{\mathbf{k}}^{\dagger}, C_{\mathbf{k}}$ are the corresponding spinors. For example, let $|\Phi\rangle$ be the ground state wave function. The resulting expectation value of the total spin current, summed up over all electrons in the gas is:

$$
\left\langle J_{j}^{a}\right\rangle=\left\langle\Phi\left|J_{j}^{a}\right| \Phi\right\rangle
$$

In the absence of special symmetry relations between the components of the Hamiltonian $H_{\mathrm{so}}$, the expectation values of spin current $\left\langle J_{j}^{a}\right\rangle$ are not zero, leading to the puzzling equilibrium spin current without any measurable spin transport. Therefore, spin current can be a characteristic of the equilibrium states of two-dimensional electron gas. In the conventional calculation of $\left\langle J_{j}^{a}\right\rangle$ due to the spin-doubling of the Fermi line, contributions to $\left\langle J_{j}^{a}\right\rangle$ come from two subsystems: single- and double occupied states at a given electron momentum. These two contributions have opposite signs and almost compensate each other, yielding the results in the third order of the coupling constants $\alpha_{j}^{3}$. This third-power dependence is expected from perturbation theory: $\left\langle J_{j}^{a}\right\rangle$ should be an odd function of the spin-orbit coupling and vanish in the first order since in the ground state without spin-orbit coupling the Fermi-line is not spin-split and all states with given $\mathbf{k}$ are doubly occupied.

Another important feature of the electron gas with spin-orbit coupling is the spin relaxation. Assume that one has initially produced a nonequilibrium state $\Phi_{S}$ of a uniform spin density with the components:

$$
S^{a}=\left\langle\Phi_{S}\left|\sum_{\mathbf{k}} C_{\mathbf{k}}^{\dagger} \tau^{a} C_{\mathbf{k}}\right| \Phi_{S}\right\rangle
$$

Then, the state $\left|\Phi_{S}\right\rangle$ will relax to the equilibrium through all possible interactions and spinorbit coupling. The first stage of the process, the momentum relaxation, is fast. If the spin-orbit coupling is weak compared to the random interactions causing the momentum relaxation, as it is assumed for the rest of this paper, the following spin relaxation is relatively slow. As a result, at the second stage the spin components decrease with relaxation rates described by a symmetric tensor $\Gamma^{a b}$ :

$$
\frac{d S^{a}}{d t}=-\Gamma^{a b} S^{b}
$$


The components of $\Gamma^{a b}$ depend on the spin-orbit coupling and all possible interactions of electrons with disorder, phonons, and other electrons in the system. [32, 33] If the spin-orbit coupling vanishes, $\Gamma^{a b}=0$.

\section{SPIN-ORBIT COUPLING AS A GAUGE FIELD: PURE GAUGE.}

Now we write the Hamiltonian of two-dimensional electron gas in the presence of spinorbit coupling as:

$$
H=\frac{1}{2 m} \int d x d y \Psi^{+}\left(i \partial_{i}+\mathcal{A}_{i}\right)^{2} \Psi+W\left(\Psi^{+}, \Psi\right)
$$

where $W\left(\Psi^{+}, \Psi\right)$ contains all explicitly spin-independent terms, including electron-electron interactions and possibly, the effect of the external potential. The general non-Abelian two-component potential is given by $2 \times 2$ matrices:

$$
\mathcal{A}_{j} \equiv A_{j}^{a} \tau^{a}=2 m \alpha_{j}(\boldsymbol{\rho}) h_{[j]}^{a}(\boldsymbol{\rho}) \tau^{a} .
$$

The expression (9) is valid for any arbitrary nonuniform spin-orbit coupling. Let us now perform at a given $\boldsymbol{\rho}$-point a local $S U(2)$-rotation [27] in the spin subspace by

$$
\mathbf{U}=\exp \left[i \theta^{a}(\boldsymbol{\rho}) \tau^{a}\right]
$$

with the transformation of the field operators:

$$
\widetilde{\Psi}^{+} \mathbf{U}^{-1}=\Psi^{+}, \quad \widetilde{\Psi}=\mathbf{U} \Psi
$$

This transformation renders the spin-independent quantities such as the charge density and the charge current density, invariant. In contrast, the spin density operators,

$$
\mathcal{S}=S^{a} \tau^{a}
$$

transforming as

$$
\mathcal{S}=\mathbf{U} \widetilde{\mathcal{S}} \mathbf{U}^{-1}
$$

exemplify covariant observable quantities. This difference between the physical quantities which transform invariantly and covariantly under a local $S U(2)$ rotation is crucial for the understanding of the spin dynamics. 
For the matrix $\mathbf{U}=\exp [i \theta(\mathbf{h} \cdot \boldsymbol{\tau})]$, where $\mathbf{h}$ is a unit length vector, the $\tau^{b}$-matrices, transformed according to Eq.(13), acquire the form:

$$
\widetilde{\tau}^{b}=h^{b}(\mathbf{h} \cdot \boldsymbol{\tau})+\cos \theta\left[\tau^{b}-h^{b}(\mathbf{h} \cdot \boldsymbol{\tau})\right]+\sin \theta \varepsilon^{a b c} h^{a} \tau^{c},
$$

where $\varepsilon^{a b c}$ is the Levi-Civita tensor. This equation shows that the product $\mathbf{h} \cdot \boldsymbol{\tau}$ is unaffected by the transformation (10). Therefore, if we present $\mathcal{S}$ as the sum of longitudinal and transverse components $\mathcal{S}=\mathcal{S}_{\|}+\mathcal{S}_{\perp}$ with $\mathcal{S}_{\|}=\mathbf{h}(\mathcal{S} \cdot \mathbf{h})$, the longitudinal component (spin projection onto the $\mathbf{h}$-axis) remains constant, while the $\mathcal{S}_{\perp}$ does not; it rotates by the angle $\theta$ around the $\mathbf{h}$-axis). This simple observation will be important for the further analysis in this paper.

The Hamiltonian preserves its form under a local $S U(2)$ rotation of the fermionic fields if the vector-potential is transformed as follows

$$
\widetilde{\mathcal{A}}_{i}=\mathbf{U}^{-1}\left(i \partial_{i} \mathbf{U}\right)+\mathbf{U}^{-1} \mathcal{A}_{i} \mathbf{U}
$$

Indeed, after the transformation the Hamiltonian acquires the form:

$$
H=\frac{1}{2 m} \int d x d y \widetilde{\Psi}^{+}\left(i \partial_{i}+\widetilde{\mathcal{A}}_{i}\right)^{2} \widetilde{\Psi}+W\left(\widetilde{\Psi}^{+}, \widetilde{\Psi}\right)
$$

which is identical to that of Eq. (8) , but with $\Psi$ and $\mathcal{A}_{i}$ being replaced by the transformed quantities, $\widetilde{\Psi}$ and $\widetilde{\mathcal{A}}_{i}$, respectively.

Assume now that $\mathcal{A}_{i}$ in the original Hamiltonian (8) corresponds to a pure gauge vectorpotential, that is both $\mathcal{A}_{x}$ and $\mathcal{A}_{y}$ can be removed by the above transformation such that $\widetilde{\mathcal{A}}_{x}=\widetilde{\mathcal{A}}_{y}=0$. In this case there exists a local rotation determined by three coordinatedependent functions $\theta_{\mathcal{A}}^{a}(x, y)$ :

$$
\mathbf{U}_{\mathcal{A}}=\exp \left[i \theta_{\mathcal{A}}^{a}(\boldsymbol{\rho}) \tau^{a}\right]
$$

such that the initial components $\mathcal{A}_{i}$ can be presented in the form

$$
\mathcal{A}_{i}=\mathbf{U}_{\mathcal{A}}\left(i \partial_{i} \mathbf{U}_{\mathcal{A}}^{-1}\right)
$$

A vector-potential of this form is gauged away by the transformation (10) with $\mathbf{U}=\mathbf{U}_{\mathcal{A}}$ :

$$
\widetilde{\mathcal{A}}_{i}=\mathbf{U}_{\mathcal{A}}^{-1}\left(i \partial_{i}+\mathbf{U}_{\mathcal{A}}\left(i \partial_{i} \mathbf{U}_{\mathcal{A}}^{-1}\right)\right) \mathbf{U}_{\mathcal{A}}=0
$$

If the spin-orbit field can be removed by a gauge transformation, the subsequent spin dynamics is simplified considerably and in certain regimes, like the drift-diffusion processes 
considered below, the problem becomes elementary. The inverse $S U(2)$-rotation transforms the spin components to the actual values and we recover the full dynamics of the physical spin. We will follow this procedure in the present paper.

We mention a textbook example of a similar approach. When the motion of a relativistic electron in static perpendicular electric field $\mathbf{E}$ and magnetic field $\mathbf{H}$ is considered, there exists a reference frame, where, after the Lorentz transformation, the smaller of these fields vanishes. In this frame the equations of the electron motion are very simple, and in the case $H<E$, they are essentially, one-dimensional. The inverse Lorentz transformation provides the full description of the electron motion in the presence of both fields. [34]

In the pure gauge field after the local $S U(2)$ transformation $\mathbf{U}_{\mathcal{A}}=\exp \left[i \theta_{\mathcal{A}}^{a}(\boldsymbol{\rho}) \tau^{a}\right]$ the Hamiltonian takes the form:

$$
H=-\frac{1}{2 m} \int d x d y \widetilde{\Psi}^{+} \Delta \widetilde{\Psi}+W\left(\widetilde{\Psi}^{+}, \widetilde{\Psi}\right)
$$

with no spin-orbit coupling present. As mentioned above, the spin dynamics with this Hamiltonian can be formulated in general terms phenomenologically and then by inverse transformation, returned to the form where the coupling and full spin dynamics are restored.

Vector-potential is a pure gauge, allowing removal six terms in $\mathcal{A}_{x}, \mathcal{A}_{y}$, with the transformation $\mathbf{U}_{\mathcal{A}}$ based on the three functions $\theta_{\mathcal{A}}^{a}(\boldsymbol{\rho})$ given certain relations between the $\mathcal{A}_{x}$ and $\mathcal{A}_{y}$ components. The corresponding conditions are naturally formulated in terms of a non-Abelian field strength tensor $\mathcal{F}_{i j}$ : the vector potential is locally a pure gauge if the field strength is zero,

$$
\mathcal{F}_{i j}=\partial_{i} \mathcal{A}_{j}-\partial_{j} \mathcal{A}_{i}-i\left[\mathcal{A}_{i}, \mathcal{A}_{j}\right]=0
$$

For the spatially uniform case, using Eq.(9) this condition is reduced to $\left[\mathcal{A}_{i}, \mathcal{A}_{j}\right]=0$, that is:

(i) either $\alpha_{i} \alpha_{j}=0$, or

(ii) $\left[\mathbf{h}_{[i]} \cdot \boldsymbol{\tau}, \mathbf{h}_{[j]} \cdot \boldsymbol{\tau}\right]=0$ if $\alpha_{i} \alpha_{j} \neq 0$.

The commutation relation

$$
\left[\mathbf{h}_{[i]} \boldsymbol{\tau}, \mathbf{h}_{[j]} \boldsymbol{\tau}\right]=i \boldsymbol{\tau} \cdot\left(\mathbf{h}_{[i]} \times \mathbf{h}_{[j]}\right)
$$

demonstrates that the spin projections commute only for the same axis, that is $\mathbf{h}_{[i]}= \pm \mathbf{h}_{[j]}$. Therefore, the solution to Eq. (22) has the form (we assume below $\mathbf{h}_{[i]}=\mathbf{h}_{[j]}$ in the case (ii) 


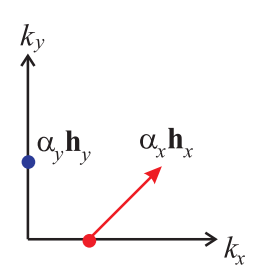

(a)

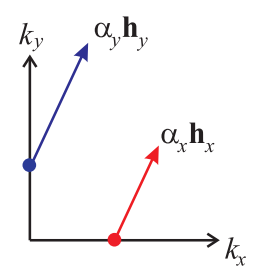

(b)

FIG. 1: (Color online) Illustration of two cases of the pure gauge spin-orbit field. (a) one of the coupling constants $\alpha_{j}$ is zero, case (i) (b) both coupling constants are not zero, the directions of corresponding magnetic fields coincide, case (ii). The direction of the spin-orbit field remains constant for any electron momentum $\mathbf{k}=\left(k_{x}, k_{y}\right)$.

for definiteness):

$$
\mathcal{A}_{j}=2 m \alpha \nu_{j}(\mathbf{h} \cdot \boldsymbol{\tau})
$$

where $\mathbf{h}=\mathbf{h}_{[i]}=\mathbf{h}_{[j]}$ if $\alpha_{i} \alpha_{j} \neq 0$ or $\mathbf{h}=\mathbf{h}_{[f]}$ for nonzero $\alpha_{f}$, where $f=x$ or $f=y$, as illustrated in Fig.(1). Here $\alpha=\left(\alpha_{x}^{2}+\alpha_{y}^{2}\right)^{1 / 2}$, and $\boldsymbol{\nu}$ is a unit vector. The corresponding gauge transformation is:

$$
\mathbf{U}_{\mathcal{A}}=\exp \left[2 i m \alpha \rho_{j} \nu_{j}(\mathbf{h} \cdot \boldsymbol{\tau})\right] \exp \left[2 i m \alpha \rho_{i} \nu_{i}(\mathbf{h} \cdot \boldsymbol{\tau})\right]=\exp [2 i m \alpha(\mathbf{h} \cdot \boldsymbol{\tau})(\boldsymbol{\rho} \cdot \boldsymbol{\nu})]
$$

From this condition we immediately conclude that the projection of the total spin at the $\mathbf{h}_{[i]}=\mathbf{h}_{[j]}$ axis commutes with $H_{\text {so }}$, and, therefore, remains constant with time for arbitrary dynamics. Experimentally, this fact corresponds to the vanishing relaxation for this spin direction; this conclusion crucial for the design and application of spin-based devices. If $\alpha_{i} \alpha_{j}=0$, the problem immediately becomes one-dimensional, trivial from the diamagnetic response interpretation of the equilibrium spin current, [27] since one-dimensional systems do not demonstrate this kind of response. The same situation occurs in quantum wires, where the motion of electrons is strictly one-dimensional, no equilibrium spin current exists, and the spin projection along the $\mathbf{h}_{[f]}$ axis is conserved. In the Appendix, for illustration, we perform a conventional calculation of the equilibrium spin current in a two-dimensional electron gas with the pure gauge spin-orbit coupling and in a quantum wire, and demonstrate that the spin current vanishes in both systems.

There are two widely studied realizations of the above discussed pure gauge field. The $\alpha_{i} \alpha_{j}=0$ case is realized for the Dresselhaus model for the electron gas confined in the quantum wells of GaAs grown along the [110] crystal axis. The coupling constant $\alpha$ in this 


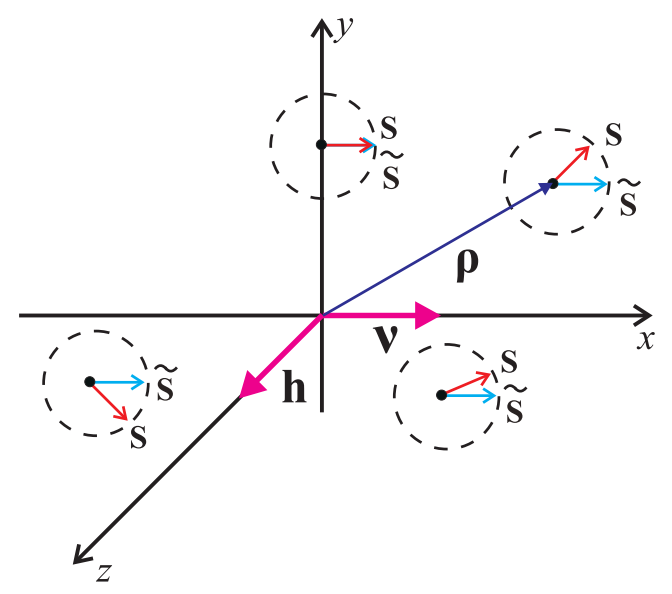

FIG. 2: (Color online) Illustration of coordinate-dependent mutual orientation of $\widetilde{\mathbf{S}}$ and $\mathbf{S}$ vectors corresponding to Eq.(26) for a structure grown along the [110] crystal axis. Vectors $\mathbf{h}$ and $\boldsymbol{\nu}$ are shown in the Figure. The angle between $\widetilde{\mathbf{S}}$ and $\mathbf{S}$ is determined solely by the $x$-component of $\rho$-vector.

system [35] is approximately inversely proportional to the square of the quantum well width $w$. In this case the vector-potential and the corresponding transformations are:

$$
\left(\mathcal{A}_{x}, \mathcal{A}_{y}\right)=\left(2 m \alpha \tau^{z}, 0\right), \quad \mathbf{U}_{\mathcal{A}}=\exp \left[2 i m \alpha x \tau^{z}\right]
$$

where the $z$ - axis is oriented along the growth direction and the $x$-axis is that of the unit cell. Here we use transformation (24) with $\mathbf{h}=(0,0,1), \boldsymbol{\nu}=(1,0)$, and $\theta(x, y)=2 m \alpha x$ to obtain:

$$
\tilde{\tau}^{z}=\tau^{z}, \quad \widetilde{\tau}^{x}=\cos \theta \tau^{x}+\sin \theta \tau^{y}, \quad \tilde{\tau}^{y}=\cos \theta \tau^{y}-\sin \theta \tau^{x}
$$

We illustrate the resulting relations between $\mathbf{S}$ and $\widetilde{\mathbf{S}}$ for this simple situation in Fig.(2): when $\widetilde{\mathbf{S}}$ remains constant in space, $\mathbf{S}$ turns by the angle $\theta(x, y)$ around the $z$-axis.

The $\alpha_{i} \alpha_{j} \neq 0$ case is realized in the compensated Dresselhaus-Rashba model for the GaAs structure grown along the [001] crystal axis. Here

$$
\begin{aligned}
\left(\mathcal{A}_{x}, \mathcal{A}_{y}\right) & =\left(2 m \alpha\left(\tau^{x}-\tau^{y}\right), 2 m \alpha\left(\tau^{x}-\tau^{y}\right)\right) \\
\mathbf{U}_{\mathcal{A}} & =\exp \left[2 i m \alpha(x+y)\left(\tau^{x}-\tau^{y}\right)\right]
\end{aligned}
$$

Here we obtain with $\mathbf{h}=(1,-1,0) / \sqrt{2}, \boldsymbol{\nu}=(1,1) / \sqrt{2}$, and $\theta=2 \sqrt{2} m \alpha(x+y)$ :

$$
\begin{aligned}
& \tilde{\tau}^{z}=\cos \theta \tau^{z}-\frac{1}{\sqrt{2}} \sin \theta\left(\tau^{x}+\tau^{y}\right) \\
& \tilde{\tau}^{x}=\cos ^{2} \frac{\theta}{2} \tau^{x}-\sin ^{2} \frac{\theta}{2} \tau^{y}+\frac{1}{\sqrt{2}} \sin \theta \tau^{z}, \quad \tilde{\tau}^{y}=\cos ^{2} \frac{\theta}{2} \tau^{y}-\sin ^{2} \frac{\theta}{2} \tau^{x}+\frac{1}{\sqrt{2}} \sin \theta \tau^{z} .
\end{aligned}
$$


Equations (26), (29) illustrate a general feature of the relations between the original $\mathbf{S}=$ $\left(S^{x}, S^{y}, S^{z}\right)$ and gauge-transformed $\widetilde{\mathbf{S}}$ spin densities and vice versa. For the spin-orbit field characterized by the direction $\mathbf{h}$, for a uniform coordinate-independent $\mathbf{S}$, the components $\mathbf{S}_{\|}$and $\widetilde{\mathbf{S}}_{\|}$coincide. The $\widetilde{\mathbf{S}}_{\perp}$-component forms a periodic structure on the spatial scale of the

order of $L_{\mathrm{so}}=1 / m \alpha$, or $\hbar^{2} / m \alpha$ when the units are restored. The mean value $\left\langle\widetilde{\mathbf{S}}_{\perp}(x, y)\right\rangle=0$ for the infinitely large systems considered here, where the boundary conditions do not change the spin dynamics. The meaning of the length $L_{\text {so }}$ can be understood as follows. Hamiltonian (2) shows that the spin-orbit coupling $H_{\text {so }}$ causes for an electron moving with the velocity $\mathbf{v}$, spin precession around $\mathbf{h}$ with the rate of the order of $\alpha m v$. The corresponding precession angle is of the order of $\alpha m L$, where $L=v t$ is the electron displacement. Thefore, $L_{\mathrm{so}}$ can be viewed as the travel distance at which the electron spin can undergo a full rotation. Another circumstance is, however, more important: the spin rotation angle depends only on the electron displacement and not on the details of its motion between initial and final points, leading to the appearance of stable spin structures, discussed below. Here a numerical value of typical $L_{\text {so }}$ can be of interest. For GaAs with $m=0.067 m_{0}$, where $m_{0}$ is the free electron mass, and $\alpha$ of the order of $10^{-7} \mathrm{meV} \cdot \mathrm{cm}, L_{\mathrm{so}}$ is of the order of several microns.

In both these systems, the observed spin relaxation rate is strongly anisotropic with one spin component having lifetime orders of magnitude longer than the others. The weak relaxation rate for these components is determined by the mechanisms different from the homogeneous spin-orbit coupling, most probably, related to the disorder in the spin-orbit coupling. [36 38$]$

\section{SPIN DYNAMICS: DIFFUSION, PRECESSIONAL BEHAVIOR, AND DRIFT CONTRIBUTIONS.}

After the gauge transformation, the spin-orbit interactions is switched off. Therefore, on a time scale much longer than the momentum relaxation time, the spin dynamics becomes combination of pure spin diffusion and spin drift:

$$
\partial_{t} \widetilde{\mathcal{S}}=D \Delta \widetilde{\mathcal{S}}+\mu E_{j} \partial_{j} \widetilde{\mathcal{S}}
$$

where $D$ is the spin-diffusion coefficient, $\mu$ is the electron mobility, and $\mathbf{E}$ is the twodimensional applied electric field [39] as illustrated in Fig.(3). In Eq.(30) we have taken 


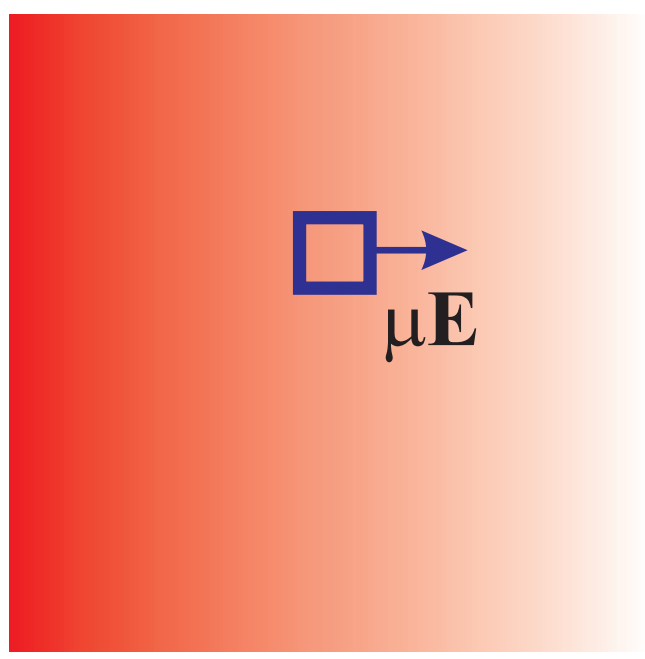

FIG. 3: (Color online) Nonuniform spin density evolving by diffusion and drift dynamics. Small square with an arrow illustrates the effect of the external electric field on the nonuniform spin dynamics.

into account that the uniform velocity of electrons is $-\mu \mathbf{E}$. These two parameters fully describe the drift-diffusive spin dynamics in the absence of spin-orbit coupling. Macroscopic motion of electrons (electric currents) can drag nonuniform spin density between different parts of the electron gas. This effect leads to the $\mu E_{j} \partial_{j} \widetilde{\mathcal{S}}$ term in $\partial_{t} \widetilde{S}$. The initial spin density eventually vanishes due to diffusion, however, the total integrated spin polarization will remain constant. The diffusive evolution of the transformed spin density $D \Delta \widetilde{\mathcal{S}}$ occurs if the electron free path of the order of $\ell=v \tau_{p}$ is much less than the spatial scale of the inhomogeneity: $\ell \ll L_{\mathrm{so}}$. This condition can be formulated as $\Omega_{\mathrm{so}} \tau_{p} \ll 1$, meaning that the spin-orbit coupling is relatively weak. The spatial inhomogeneity of the order of $L_{\text {so }}$ and $D$ of the order of $v^{2} \tau_{p}$ set the time scale of the diffusion smearing of the $\widetilde{\mathcal{S}}$ as $\widetilde{t}_{D} \sim L_{\text {so }}^{2} / D$ on the order of $\Omega_{\text {so }}^{-2} \tau_{p}^{-1}$, and, therefore, the same spin relaxation time for real spin $\mathcal{S}$.

The evolution of the physical measurable spin density:

$$
\mathcal{S}=\mathbf{U}_{\mathcal{A}} \widetilde{\mathcal{S}} \mathbf{U}_{\mathcal{A}}^{-1}
$$

is due to the diffusion and spin precession since the transition of electron from point $\boldsymbol{\rho}_{1}$ to point $\boldsymbol{\rho}_{2}$ is accompanied by the rotation of its spin, dependent only on the displacement $\boldsymbol{\rho}_{2}-\boldsymbol{\rho}_{1}$. Irregular motion in the diffusion process is seen in the spin relaxation, and regular drift causes spin precession, with these two processes being mutually related.

Motion of $\mathcal{S}$ is described, therefore, by the following equations for the time evolutions 
of the spin density, which are obtained by applying the inverse transformation (31) to the drift-diffusion equation (30),

$$
\partial_{t} \mathcal{S}=D \mathbf{U}_{\mathcal{A}}\left[\Delta\left(\mathbf{U}_{\mathcal{A}}^{-1} \mathcal{S} \mathbf{U}_{\mathcal{A}}\right)\right] \mathbf{U}_{\mathcal{A}}^{-1}+\mu E_{j} \mathbf{U}_{\mathcal{A}}\left[\partial_{j}\left(\mathbf{U}_{\mathcal{A}}^{-1} \mathcal{S} \mathbf{U}_{\mathcal{A}}\right)\right] \mathbf{U}_{\mathcal{A}}^{-1}
$$

The resulting most general equation of motion valid for any pure gauge spin-orbit field takes the form

$$
\begin{aligned}
& \partial_{t} \mathcal{S}-D \Delta \mathcal{S}-\mu E_{j} \partial_{j} \mathcal{S} \\
= & D\left\{2\left[\mathbf{U}_{\mathcal{A}} \nabla \mathbf{U}_{\mathcal{A}}^{-1}, \nabla \mathcal{S}\right]-2\left(\mathbf{U}_{\mathcal{A}} \nabla \mathbf{U}_{\mathcal{A}}^{-1}\right) \mathcal{S}\left(\mathbf{U}_{\mathcal{A}} \nabla \mathbf{U}_{\mathcal{A}}^{-1}\right)+\left(\mathbf{U}_{\mathcal{A}} \Delta \mathbf{U}_{\mathcal{A}}^{-1}\right) \mathcal{S}+\mathcal{S}\left(\Delta \mathbf{U}_{\mathcal{A}}\right) \mathbf{U}_{\mathcal{A}}^{-1}\right\} \\
& +\mu E_{j}\left[\left(\mathbf{U}_{\mathcal{A}} \partial_{j} \mathbf{U}_{\mathcal{A}}^{-1}\right), \mathcal{S}\right] .
\end{aligned}
$$

The total expression for local evolution of spin density components can be obtained from this equation by multiplying both sides by $\tau^{a}$ and taking the trace using the identity: $\operatorname{tr}\left(\tau^{a} \tau^{b}\right)=$ $\delta^{a b} / 2$. The result can be presented as the sum:

$$
\partial_{t} S^{a}=D \Delta S^{a}+\mu E_{j} \partial_{j} S^{a}+B_{j}^{a b} \partial_{j} S^{b}-H^{a b} S^{b}-\Gamma^{a b} S^{b}
$$

The general expressions for non-diagonal tensors of kinetic coefficients entering this equation are:

$$
\begin{aligned}
B_{j}^{a b} & =-B_{j}^{b a}=4 D \operatorname{tr}\left\{\tau^{a}\left[\mathbf{U}_{\mathcal{A}} \partial_{j} \mathbf{U}_{\mathcal{A}}^{-1}, \tau^{b}\right]\right\} \\
H^{a b} & =-H^{b a}=2 \mu E_{j} \operatorname{tr}\left\{\tau^{a}\left[\mathbf{U}_{\mathcal{A}} \partial_{j} \mathbf{U}_{\mathcal{A}}^{-1}, \tau^{b}\right]\right\} \\
\Gamma^{a b} & =4 D\left(\operatorname{tr}\left\{\tau^{a}\left(\mathbf{U}_{\mathcal{A}} \partial_{j} \mathbf{U}_{\mathcal{A}}^{-1}\right) \tau^{b}\left(\mathbf{U}_{\mathcal{A}} \partial_{j} \mathbf{U}_{\mathcal{A}}^{-1}\right)\right\}-\frac{1}{2} \operatorname{tr}\left\{\tau^{a}\left(\mathbf{U}_{\mathcal{A}} \Delta \mathbf{U}_{\mathcal{A}}^{-1}\right) \tau^{b}+\tau^{a}\left(\Delta \mathbf{U}_{\mathcal{A}}\right) \mathbf{U}_{\mathcal{A}}^{-1} \tau^{b}\right\}\right)
\end{aligned}
$$

Now we can study the physical meaning of the obtained non-diagonal tensors and simplify the expressions for the time derivatives for uniform spin-orbit coupling with:

$$
\mathbf{U}_{\mathcal{A}}=\exp [2 i m \alpha(\mathbf{h} \cdot \boldsymbol{\tau})(\boldsymbol{\nu} \cdot \boldsymbol{\rho})], \quad \mathbf{U}_{\mathcal{A}}^{-1}=\mathbf{U}_{\mathcal{A}}^{+}=\exp [-2 i m \alpha(\mathbf{h} \boldsymbol{\tau})(\boldsymbol{\nu} \cdot \boldsymbol{\rho})],
$$

With the given form in Eq. (38) of $\mathbf{U}_{\mathcal{A}}$ and $\mathbf{U}_{\mathcal{A}}^{-1}$ we obtain:

$$
\mathbf{U}_{\mathcal{A}} \nabla \mathbf{U}_{\mathcal{A}}^{-1}=-2 i m \alpha(\mathbf{h} \cdot \boldsymbol{\tau}) \boldsymbol{\nu}, \quad \Delta \mathbf{U}_{\mathcal{A}}=-4 m^{2} \alpha^{2} \mathbf{U}_{\mathcal{A}}, \quad \Delta \mathbf{U}^{-1}=-4 m^{2} \alpha^{2} \mathbf{U}_{\mathcal{A}}^{-1}
$$

With formulas (38), (39) we obtain for the diffusion-related coefficients:

$$
\begin{aligned}
B_{j}^{a b} & =-2 m \alpha_{j} D \varepsilon^{a b c} h^{c}, \\
\Gamma^{a b} & =4 m^{2} \alpha^{2} D\left(\delta^{a b}-h^{a} h^{b}\right) .
\end{aligned}
$$


The corresponding drift-dependent contribution:

$$
H^{a b}=-m \alpha \mu(\boldsymbol{\nu} \cdot \mathbf{E}) \varepsilon^{a b c} h^{c},
$$

describes the spin precession.

The answer for the spin density $\mathbf{S}$ with components $\left(S^{x}, S^{y}, S^{z}\right)$ has the form of three terms of different order in $\alpha$ :

$$
\partial_{t} \mathbf{S}=\left.\partial_{t} \mathbf{S}\right|_{0}+\left.\partial_{t} \mathbf{S}\right|_{1}+\left.\partial_{t} \mathbf{S}\right|_{2}
$$

These terms have different meaning and can be expressed as:

$$
\begin{aligned}
& \left.\partial_{t} \mathbf{S}\right|_{0}=D \Delta \mathbf{S}+\mu E_{j} \partial_{j} \mathbf{S}, \\
& \left.\partial_{t} \mathbf{S}\right|_{1}=4 m D \alpha(\boldsymbol{\nu} \cdot \nabla)(\mathbf{h} \times \mathbf{S})+2 m \alpha \mu(\boldsymbol{\nu} \cdot \mathbf{E})(\mathbf{h} \times \mathbf{S}), \\
& \left.\partial_{t} \mathbf{S}\right|_{2}=-4 m^{2} \alpha^{2} D(\mathbf{S}-\mathbf{h}(\mathbf{h} \cdot \mathbf{S})) .
\end{aligned}
$$

The $\left.\partial_{t} \mathbf{S}\right|_{0}$ term describes the standard drift-diffusion spin dynamics for zero spin-orbit coupling.

The $\left.\partial_{t} \mathbf{S}\right|_{1}$ term corresponds to the spin precession due to the spin-orbit coupling. The mobility-determined contribution in $\left.\partial_{t} \mathbf{S}\right|_{1}$ is the precession in the macroscopic spin-orbit field arising due to the uniform velocity of electrons. When the electric current is induced, the momentum distribution function is shifted such that the momentum has a nonzero value. As a result, the Hamiltonian $H_{\text {so }}$ forms a macroscopic spin-orbit Zeeman field [40] and, as a result, a regular spin precession $\partial_{t} \mathbf{S}=2 m \alpha \mu(\boldsymbol{\nu} \cdot \mathbf{E})(\mathbf{h} \times \mathbf{S})$. If $(\boldsymbol{\nu} \cdot \mathbf{E})=0$, contributions of the momentum changes along the $x$ and $y$-axes in the macroscopic spin-orbit "magnetic" field compensate each other, and no regular precession occurs. Thus, Eq.(45) reproduces the diffusive and non-diffusive spin precession.

The $\left.\partial_{t} \mathbf{S}\right|_{2}$ term is the Dyakonov-Perel' mechanism of spin relaxation, [41] which can be seen from the fact that $D$ is determined by $\left\langle v^{2}\right\rangle \tau_{p}$, where $v$ is the electron velocity (see, also in Ref. [13]). Taking into account that electron momentum is $m v$, one can see that $\left.\partial_{t} \mathbf{S}\right|_{2}$ corresponds to the Dyakonov-Perel relaxation with the relaxation rate on the order of $\alpha^{2} k^{2} \tau_{p}$. The obtained relation between the spin relaxation rate and diffusion coefficient is universal. For two different systems with the same sample-dependent $m \alpha$ parameter, the ratio of $\Gamma^{a b} / D$ remains constant. Since the parameters $\Gamma^{a b}$ and $D$ can be measured independently, this universality can be verified experimentally. For example, in the measurements performed at 
the same sample at different temperatures, the ratio $\Gamma^{a b} / D$ is expected to remain constant for degenerated and non-degenerated electron gas.

Eqs.(45) and (46) show that $\mathbf{S}_{\|}=\mathbf{h} \cdot \mathbf{S}$ does not change with time, as expected, and the entire dynamics is solely due to the $\mathbf{S}_{\perp}$-component. As a simple illustration we consider the evolution of an initially homogeneous spin density. By solving equations (43)-(46) with the initial condition $\mathbf{S}(\boldsymbol{\rho}, t=0)=\mathbf{S}_{0}$ we find the spin dynamics

$$
\mathbf{S}(t)=\mathbf{h}\left(\mathbf{h} \cdot \mathbf{S}_{0}\right)+\left\{\cos \left(\Omega_{E} t\right)\left[\mathbf{S}_{0}-\mathbf{h}\left(\mathbf{h} \cdot \mathbf{S}_{0}\right)\right]+\sin \left(\Omega_{E} t\right)\left(\mathbf{h} \times \mathbf{S}_{0}\right)\right\} e^{-\Gamma t},
$$

where $\Omega_{E}=2 \alpha m \mu(\boldsymbol{\nu} \cdot \mathbf{E})$ is the precession frequency in a drift-induced spin-orbit Zeeman field, and $\Gamma=4 \alpha^{2} m^{2} D$ is the diffusion related relaxation rate. From Eq. (47) we see that the spin precesses with the frequency $\Omega_{E}$ about the $\mathbf{h}$-axis and its transverse component decays at the rate $\Gamma$ in such a way that the projection of the spin at $\mathbf{h}$ remains stationary. By comparing the characteristic time scales of the drift-induced precession and the diffusioninduced relaxation, we can estimate the external electric field at which the role of the drift-dependent terms becomes important; in particular, the precession becomes visible at the scale of the relaxation time. From the condition of still visible precession $\Omega_{E} \sim \Gamma$ we find the corresponding electric field $E \sim \alpha m D / \mu$. In this field, the precession rate $\Omega_{E}$ is of the order of $\Omega_{\mathrm{so}}^{2} \tau_{p}$, making the contributions of regular and random motion in the precession angle of the same order. If the spin diffusion is dominated by the impurity scattering, then $D$ and $\mu$ are proportional to the momentum relaxation time $\tau_{p}$, and this electric field is disorder-independent. However, it can change with the temperature since in the nondegenerated gas $D$ approaches the electron diffusion coefficient [39] and, therefore, by the Einstein relation $D=\mu T$.

Another interesting effect of a spin-orbit coupling, which follows straightforwardly from our formulation - the existence of stable spatially inhomogeneous spin configuration. It is easy to verify that a general stationary $\left(\partial_{t} \mathbf{S}=\mathbf{0}\right)$ solution to the equations (43)-(46) is of the form

$$
\mathbf{S}(\boldsymbol{\rho})=\mathbf{h}\left(\mathbf{h} \cdot \mathbf{S}_{0}\right)+\cos (2 m \alpha(\boldsymbol{\nu} \cdot \boldsymbol{\rho}))\left[\mathbf{S}_{0}-\mathbf{h}\left(\mathbf{h} \cdot \mathbf{S}_{0}\right)\right]-\sin (2 m \alpha(\boldsymbol{\nu} \cdot \boldsymbol{\rho}))\left(\mathbf{h} \times \mathbf{S}_{0}\right),
$$

where $\mathbf{S}_{0}$ is an arbitrary constant vector. This spatially inhomogeneous stationary solution to the drift-diffusion equation arises due to the symmetry of the system. As it was demonstrated for the particular case of the model with balanced Rashba and Dresselhaus couplings, the 
symmetry can protect electron spins from relaxation [29] and allows for the persistent spin helix structures [42, 43] of the form of Eq.(48). The fact that the shape of this configuration does not depend on the diffusion coefficient $D$ shows that the persistent spin structure is insensitive to the spin-independent disorder, in agreement with Ref.[42] The analysis of the spin helix stability in the presence of disordered spin-orbit coupling can be found in Ref. [44] It is interesting to note that the helix structure is also insensitive to the presence of the electric field and, therefore, to the mobility and presence of a transport charge current, at least in the linear Ohm's law regime. This seemingly counterintuitive result follows from the fact that the drift of the helix governed by the second term in (44) is exactly compensated by the spin precession in the current-induced effective Zeeman field, the second term in (45). A similar cancellation occurs in the diffusion channel. A diffusive spreadout of the helix, the first term in (44), and the relaxation of the transverse component of the spin, (46), are balanced by the "gradient-precession" contribution, the first term in (45). The persistent spin helix configuration (48) has an extremely simple interpretation in terms of the transformed spin density $\widetilde{\mathbf{S}}$. The general stationary solution of the standard drift-diffusion equation (30) is simply a constant $\widetilde{\mathbf{S}}=\widetilde{\mathbf{S}}_{0}$. The relation between the physical and transformed spin density components yield the conservation $\widetilde{\mathbf{S}}_{0} \cdot \mathbf{h}=\mathbf{S} \cdot \mathbf{h}$. The perpendicular $\widetilde{\mathbf{S}}_{0, \perp}$ is transformed according to Eq. (31) as $\mathcal{S}=\mathbf{U}_{\mathcal{A}} \widetilde{\mathcal{S}} \mathbf{U}_{\mathcal{A}}^{-1}$ with $\mathbf{U}_{\mathcal{A}}=\exp [2 i m \alpha(\mathbf{h} \cdot \boldsymbol{\tau})(\boldsymbol{\rho} \cdot \boldsymbol{\nu})]$ from Eq. (24) according to Eq. (14). The sum of the transformed terms is precisely the persistent spin helix of Eq. (48).

It is also instructive to look at the precession and relaxation of a spatially homogeneous spin $\mathbf{S}(t)$, Eq. (47), from the point of view of the dynamics of the transformed spin density $\widetilde{\mathbf{S}}$. The initial condition $\mathbf{S}(\boldsymbol{\rho}, t=0)=\mathbf{S}_{0}$ for the physical spin is mapped to the initial configuration for $\widetilde{\mathbf{S}}$ in a form of a spin helix that is similar to Eq. (48). The subsequent evolution of $\widetilde{\mathbf{S}}$ is governed by the standard drift-diffusion equation (30). Therefore the dynamical behavior is obvious -the initial helix for the transformed spin moves with the drift velocity $v_{\text {drift }}=m \alpha \mu(\boldsymbol{\nu} \cdot \mathbf{E})$, and washes out diffusively. When transformed to the physical spin, the drift of the helix translates to the precession, while its diffusive decay is mapped to the relaxation of the physical spin. This interpretation clearly explains why the relaxation time of the transverse components of the spin is universally determined by the diffusion coefficient. The relaxation of the physical spin components is gauge-equivalent to a purely diffusive process of washing out the initial helix configuration of the transformed 
spin.

\section{CONCLUSIONS.}

We developed a gauge theory of macroscopic spin dynamics in a two-dimensional electron gas when the spin-orbit coupling can be described as a pure gauge, and, therefore, removed by a local $S U(2)$ rotation in the spin subspace. We have shown that for a pure spin gauge, equilibrium spin current vanishes and a selected axis of conserved spin projection appears simultaneously, demonstrating gauge-related symmetry relation of these effects. After removing the spin-orbit coupling, we considered macroscopic phenomenological equations of spin dynamics, including spin diffusion and spin drift in an external electric field. By the inverse $S U(2)$ rotation we obtained the full system of partial differential equations for the time- and spatial measured spin density evolution. This system reproduces the physics of spin precession, stable spin configurations such as the persistent spin helix, and the resulting strongly anisotropic spin relaxation. Since we described the system without spin-orbit coupling phenomenologically, our approach is valid at any temperature and electron concentration. It predicts that the ratio of the spin relaxation rate to the spin diffusion coefficient remains temperature- and electron concentration-independent if the coupling constants do not depend on these two system parameters.

We presented explicit equations for the spatially uniform spin-orbit coupling and their solutions describing stable nonuniform structures, the precession and the relaxation of uniform spin polarization. These equations can be explicitly generalized for nonuniform twodimensional electron gas in macroscopic systems. We mention two of them. The first one is the GaAs quantum well grown along the [110] direction with a modulated width $w(x)$, where the spin-orbit $\alpha(x)$ originated from the Dresselhaus coupling, varies as $1 / w^{2}(x)$. The corresponding spin-orbit field $\mathcal{A}_{x}=2 m \alpha(x) \tau^{z}, \mathcal{A}_{y}=0$, with $\partial \mathcal{A}_{x} / \partial y=0$ remains a pure gauge. The other example is the balanced Rashba-Dresselhaus model with the coordinatedependent Rashba and Dresselhaus parameters remaining exactly equal or exactly opposite everywhere. As in the [110] structure, variation in the Dresselhaus term is due to the controlled variation in the structure width, while the control of the Rashba coupling is achieved by a coordinate-dependent bias across the well. A different kind of inhomogeneity occurs in mesoscopic systems where the effect of the boundary conditions for the coupled spin- 
charge dynamics becomes important.[45-47] Generalization of the gauge theory approach for the dynamics at the sample boundaries can be an interesting extension of our analysis for the infinite systems. Spin dynamics in these systems is of interest for the fundamental understanding of spin transport and for applications in spintronics devices.

\section{ACKNOWLEDGMENT.}

IVT acknowledges funding by the Spanish MEC (FIS2007-65702-C02-01), "Grupos Consolidados UPV/EHU del Gobierno Vasco" (IT-319-07), and the European Community through e-I3 ETSF project (Grant Agreement: 211956). EYS is grateful to the University of Basque Country UPV/EHU for support by the Grant GIU07/40.

\section{APPENDIX.}

Here we show by a conventional calculation of the spin current that it vanishes at the equilibrium in the considered above pure gauge spin-orbit coupling in a two-dimensional electron gas and, similarly, in one-dimensional quantum wires. We begin with the puregauge two-dimensional Hamiltonian,

$$
H=\frac{k_{x}^{2}}{2 m}+\alpha_{x}\left(h^{a} \sigma^{a}\right) k_{x}+\frac{k_{y}^{2}}{2 m}+\alpha_{y}\left(h^{a} \sigma^{a}\right) k_{y},
$$

where $\mathbf{h}$ is unit length vector and $\alpha_{x}, \alpha_{y}$ are the corresponding spin-orbit coupling constants. The spectrum of electrons described by Eq.(49) is the sum of $k_{x}$ and $k_{y}$-dependent terms for the two spin branches "+ " and "-":

$$
\varepsilon_{ \pm}\left(k_{x}, k_{y}\right)=\frac{k_{x}^{2}+k_{y}^{2}}{2 m} \pm\left(\alpha_{x} k_{x}+\alpha_{y} k_{y}\right) .
$$

Eq. (49) demonstrates that the system with pure gauge spin-orbit coupling remains in a certain sense, one dimensional: spin-orbit coupling does not couple different components

of momentum in the spectrum. For illustration we consider only the $x$-component of momentum and velocity:

$$
\frac{\partial \varepsilon_{ \pm}(\mathbf{k})}{\partial k_{x}}=\frac{k_{x}}{m} \pm \alpha_{x}, \quad v_{x}=i[H, x]=\frac{k_{x}}{m}+\alpha_{x}\left(h^{a} \sigma^{a}\right)
$$




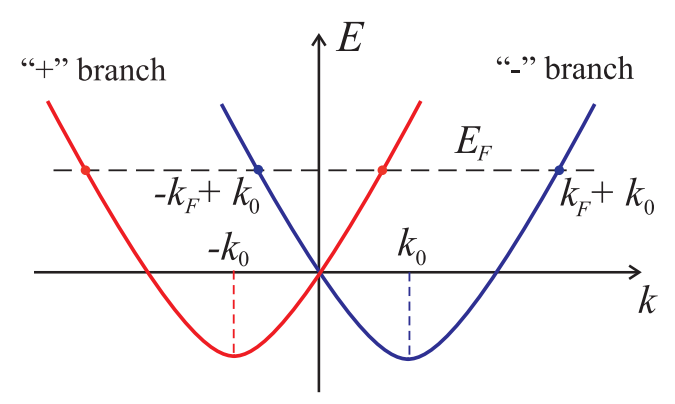

FIG. 4: (Color online) Scheme of the spin-orbit split states in a quantum wire. $E_{F}$ is the Fermi energy.

yielding the spin current component:

$$
J_{x}^{b}=\frac{1}{4} \sum_{\mathbf{k}} C_{\mathbf{k}}^{\dagger}\left\{v_{x}, \sigma^{b}\right\} C_{\mathbf{k}}
$$

Taking into account that $\left\{\sigma^{a}, \sigma^{b}\right\}=2 \delta^{a b}$, we obtain the anticommutator:

$$
\frac{1}{2}\left\{v_{x}, \sigma^{b}\right\}=\frac{1}{2}\left\{\frac{k_{x}}{m}+\alpha_{x}\left(h^{a} \sigma^{a}\right), \sigma^{b}\right\}=\frac{k_{x}}{m} \sigma^{b}+\alpha_{x} h^{b} .
$$

The total spin current is the sum of contributions of two subsystems $\left\langle J_{x}^{b}\right\rangle=\left\langle J_{x}^{b}\right\rangle_{+}+\left\langle J_{x}^{b}\right\rangle_{-}=$ $2\left\langle J_{x}^{b}\right\rangle_{+}$. Taking into account that for given branch $\left\langle\sigma^{b}\right\rangle_{ \pm}= \pm h^{b} / h$, the $\left\langle J_{x}^{b}\right\rangle_{+}$spin current component becomes:

$$
\left\langle J_{x}^{b}\right\rangle_{+}=\frac{1}{2} h^{b} \int d k_{y} \int \frac{\partial \varepsilon(\mathbf{k})}{\partial k_{x}} d k_{x}
$$

where the integration is perfromed over the area in momentum space occupied by electrons from the branch. The value of this integral is zero since this area is restricted by the line of the constant Fermi energy $E_{F}$.

For one-dimensional case the situation is the same. We take the Hamiltonian:

$$
H=\frac{k^{2}}{2 m}+\alpha\left(h^{a} \sigma^{a}\right) k .
$$

The eigenstates of this Hamiltonian form two branches:

$$
\varepsilon_{ \pm}=\frac{k^{2}}{2 m} \pm \alpha k
$$

corresponding to two parabolas with the minima at $-k_{0}$ and $k_{0}=\alpha m$, respectively, as shown in Fig.(4). To calculate the spin current directly, we perform integration over momenta and summation over spin branches. The ground state expectation value is:

$$
\left\langle J^{b}\right\rangle=\frac{1}{2}\left[\int_{-k_{0}-k_{F}}^{-k_{0}+k_{F}}\left(\frac{k}{m}\left\langle\sigma^{b}\right\rangle_{+}+h^{b}\right) d k+\int_{k_{0}-k_{F}}^{k_{0}+k_{F}}\left(\frac{k}{m}\left\langle\sigma^{b}\right\rangle_{-}+h^{b}\right) d k\right],
$$


where $k_{F}$ is the Fermi momentum determined by the total concentration of electrons $n$ as $k_{F}=\pi n / 2$. We obtain

$$
\begin{aligned}
\left\langle J^{b}\right\rangle & =2 k_{F} h^{b}+\frac{1}{2 m}\left[\int_{-k_{0}-k_{F}}^{k_{0}-k_{F}} k \frac{h^{b}}{h} d k-\int_{-k_{0}+k_{F}}^{k_{0}+k_{F}} k \frac{h^{b}}{h} d k\right] \\
& =2 k_{F} h^{b}-\frac{2}{m} \frac{h^{b}}{h} k_{F} k_{0} .
\end{aligned}
$$

The minimum position $k_{0}=\alpha m$, yields $\left\langle J^{b}\right\rangle=0$, as expected.

The absence of the spin current in a wire can be related to the vanishing persistent Aharonov-Bohm spin current [48, 49] in a ring with spin-orbit coupling as the ring radius goes to infinity. Indeed, if a ring is pierced by a small-radius solenoid with a finite magnetic field flux confined inside it, the field at the ring is a pure gauge, and an Aharonov-Bohm current in the ring arises. The spin-orbit coupling in a ring can be understood in terms of a spin-dependent gauge, and a corresponding spin current can be induced. This spin current vanishes in the $R \rightarrow \infty$ limit. In general, however, these two objects have a different topology: a ring can demonstrate a diamagnetic response, while a wire cannot. 
[1] I. Zutic, J. Fabian, S. Das Sarma, Rev. Mod. Phys. 76 (2004) 323.

[2] J. Fabian, A. Matos-Abiague, C. Ertler, P. Stano, I. Zutic, Acta Physica Slovaca 57 (2007) 565.

[3] Spin Physics in Semiconductors, Springer Series in Solid-State Sciences, Ed. by M.I. Dyakonov, Springer (2008)

[4] B.W.A. Leurs, Z. Nazario, D.I. Santiago, J. Zaanen, Annals of Physics 323 (2008) 907.

[5] P. Kleinert, V. V. Bryksin, Phys. Rev. B 79 (2009) 045317.

[6] T. D. Stanescu, and V. Galitski, Phys. Rev. B 75 (2007) 125307.

[7] E. I. Rashba, Al. L. Efros, Phys. Rev. Lett. 91 (2003) 126405.

[8] E. G. Mishchenko, A. V. Shytov, B. I. Halperin, Phys. Rev. Lett. 93 (2004) 226602.

[9] D. Culcer, R. Winkler, Phys. Rev. B, 76 (2007) 195204; D. Culcer, preprint cond-mat arXiv:0904.1999 (2009); R. Winkler, D. Culcer, S. J. Papadakis, B. Habib, M Shayegan, Semicond. Sci. Technol. 23 (2008) 114017.

[10] M. M. Glazov, Sol. State Commun. 142 (2007) 531; Phys. Rev. B 70 (2004) 195314.

[11] J. L. Cheng, M. W. Wu, C. Lü, Phys. Rev. B 69 (2004) 115318; P. Zhang, M. W. Wu, Phys. Rev. B 79 (2009) 075303

[12] F. X. Bronold, A. Saxena, D. L. Smith, Phys. Rev. B 70 (2004) 245210; F. X. Bronold, I. Martin, A. Saxena, D. L. Smith, Phys. Rev. B 66 (2002) 233206.

[13] R. Raimondi, P. Schwab, Europhys. Lett. 87 (2009) 37008; M. Milletar, R. Raimondi, P. Schwab Europhys. Lett. 82 (2008) 67005.

[14] O. Bleibaum, Phys. Rev. B 73 (2006) 035322; O. Bleibaum, Phys. Rev. B 72 (2005) 075366.

[15] E. I. Rashba, Phys. Rev. B 68 (2003) 241315.

[16] V. P. Mineev, G. E. Volovik, Journal of Low Temperature Physics 89 (1992) 823.

[17] J. Fröhlich, U. M. Studer, Rev. Mod Phys. 65, (1993) 733.

[18] I. L. Aleiner, V. I. Fal'ko, Phys. Rev. Lett. 87 (2001) 256801.

[19] L. S. Levitov, E. I. Rashba, Phys. Rev. B 67 (2003) 115324.

[20] K. C. Nowack, F. H. L. Koppens, Yu. V. Nazarov, L. M. K. Vandersypen, Science 318 (2007) 1430.

[21] J.-S. Yang, X.-G. He, S.-H. Chen, C.-R. Chang, Phys. Rev. B 78 (2008) 085312. 
[22] S.-H. Chen, C.-R. Chang, Phys. Rev. B 77 (2008) 045324.

[23] Q. Liu, T. Ma, S.-C. Zhang, Phys. Rev. B 76 (2007) 233409.

[24] S.-R. Eric Yang, Phys. Rev. B 75 (2007) 245328

[25] N. Hatano, R. Shirasaki, H. Nakamura, Phys. Rev. A 75 (2007) 032107.

[26] S.-R. Eric Yang, N. Y. Hwang, Phys. Rev. B 73 (2006) 125330.

[27] I. V. Tokatly, Phys. Rev. Lett. 101 (2008) 106601.

[28] N. S. Averkiev L. E. Golub, Phys. Rev. B 60 (1999) 15582.

[29] J. Schliemann, J. C. Egues, D. Loss, Phys. Rev. Lett. 90 (2003) 146801.

[30] Yu. A. Bychkov, E. I. Rashba, JETP Lett. 39, (1984) 79.

[31] R. Winkler, Spin-orbit Coupling Effects in Two-Dimensional Electron and Hole Systems, Springer Tracts in Modern Physics (2003).

[32] M. M. Glazov, E. L. Ivchenko, Pis'ma Zh. Eksp. Teor. Fiz. 75 (2002) 476 [JETP Lett. 75 (2002) 403]; M. M. Glazov, E. L. Ivchenko, JETP 99 (2004) 1279.

[33] D. Stich, J. Zhou, T. Korn, R. Schulz, D. Schuh, W. Wegscheider, M. W. Wu, C. Schüller, Phys. Rev. Lett. 98 (2007) 176401.

[34] J.D. Jackson Classical Electrodynamics, Third Edition, New York, Academic Press (1998).

[35] M.I. Dyakonov, V.Yu. Kachorovskii, Fiz. Tekh. Poluprovodn. (St.-Petersburg) 20 (1986) 178, [Sov. Phys. Semicond. 20, (1986) 110].

[36] G. M. Müller, M. Römer, D. Schuh, W. Wegscheider, J. Hübner, M. Oestreich, Phys. Rev. Lett. 101 (2008) 206601.

[37] M. M. Glazov, E. Ya. Sherman, Phys. Rev. B 71 (2005) 241312(R).

[38] V. K. Dugaev, E. Ya. Sherman, V. I. Ivanov, J. Barnaś, Phys. Rev. B 80 (2009) 081301.

[39] I.D'Amico, G. Vignale, Europhys. Lett. 55(2001) 566.

[40] Z. Wilamowski, W. Ungier, W. Jantsch, Phys. Rev. B 78 (2008) 174423.

[41] M.I. Dyakonov, V.I. Perel', Sov. Phys. Solid State 13 (1972) 3023.

[42] B. A. Bernevig, J. Orenstein, S.-C. Zhang, Phys. Rev. Lett. 97 (2006) 236601.

[43] J. D. Koralek, C. P. Weber, J. Orenstein, B. A. Bernevig, Shou-Cheng Zhang, S. Mack, D. D. Awschalom, Nature 458 (2009) 610.

[44] M.-H. Liu, K.-W. Chen, S.-H. Chen, C.-R. Chang, Phys. Rev. B 74 (2006) 235322.

[45] Y. Tserkovnyak, B. I. Halperin, A. A. Kovalev, A. Brataas, Phys. Rev. B 76 (2007) 085319.

[46] O. Bleibaum, Phys. Rev. B 74 (2006) 113309. 
[47] M. Duckheim, D. L. Maslov, D. Loss, Phys. Rev. B 80, (2009) 235327.

[48] R. Citro, F. Romeo, Phys. Rev. B 75 (2007) 073306.

[49] Q.-F. Sun, X. C. Xie, J. Wang, Phys. Rev. Lett. 98, 196801 (2007); Q.-F. Sun, X. C. Xie, J. Wang, Phys. Rev. B 77 (2008) 035327. 